\title{
Internal Motions in the Orion Nebula*
}

\author{
G. $\mathrm{MÜNCH}$ \\ Mount Wilson and Palomar Observatories, Carnegie Institution of Washington, California \\ Institute of Technology, Pasadena, California
}

$I^{\mathrm{N}}$ the first and second Symposia of this series von Weizsäcker ${ }^{1}$ and von Hoerner ${ }^{2}$ discussed the problem of turbulence in the Orion Nebula, while in the second Symposium Courtès ${ }^{3}$ has further treated the problem. Von Hoerner ${ }^{4}$ has presented a detailed discussion of the methodologies of the treatment. It was suggested that the observed variations in radial velocity in the nebula are consistent with the predictions of the Kolmogoroff equilibrium theory of turbulence, which is valid at sufficiently high Reynolds numbers. However, their results to some extent were inconclusive, mainly because the observations which they analyzed were not sufficiently numerous and accurate. With the purpose of reanalyzing the whole problem, Dr. O. C. Wilson and $I$ undertook the task of determining radial velocities and profiles of selected emission lines in the spectrum of the nebula, using the largest practical resolving power in angle and frequency available with the 200-in. telescope. In order to use advantageously the efficiency of the instrument, we have photographed the brighter parts of the nebula (roughly subtending a solid angle of about $6^{\prime}$ aperture) with the Coudé spectrograph fitted with 31 parallel entrance slits, which are separated from each other by a distance of $1 \mathrm{~mm}$ in the focal plane or $1^{\prime \prime} .3$ in the sky. In this manner we obtain in one exposure the spectrum of an area about $40^{\prime \prime} \times 40^{\prime \prime}$ with a dispersion such that $1 \mu=0.27 \mathrm{~km} / \mathrm{sec}$. In each of these plates about 600 Doppler shifts of the lines $[\mathrm{OII}] \lambda 3726, \mathrm{H} \gamma$, and [OII] $\lambda 5007$ have been measured, each of which represents some average value (not necessarily the same for the three lines) of the velocities of nebular matter along the line of sight. Altogether we have about 50000 radial velocities measured. The accuracy with which a radial velocity may be determined is set by the intrinsic shape of the lines, which reflects the distribution of velocities along the line of sight. To give an idea of the orders of magnitude of the quantities involved, I may mention here that typical values of the mean widths $h$ at halfintensity of the hydrogen, [OIII], and $\mathrm{Fe}$-comparison

\footnotetext{
* Supported in part by the Office of Naval Research under contract Nonr-1668(00) with the California Institute of Technology.

1 C. F. von Weiszäcker, "Problems of cosmical aerodynamics," C.A.D.O. Dayton, Ohio, (1951), Chap. 22.

${ }^{2}$ S. von Hoerner, Gas Dynamics of Cosmic Clouds, edited by H. C. van de Hulst and J. M. Burgers (North Holland Publishing Company, Amsterdam, 1955), Chap. 32.

${ }_{3}$ G. Courtès, Gas Dynamics of Cosmic Clouds, edited by H. C. van de Hulst and J. M. Burgers (North Holland Publishing Company; Amsterdam, 1955), Chap. 23.

${ }^{4}$ S. von Hoerner, Z. Astrophys. 30, 17 (1951).
}

lines are $h(\mathrm{H})=28.6 \mathrm{~km} / \mathrm{sec}, h(\mathrm{OIII})=20.0 \mathrm{~km} / \mathrm{sec}$, $h(\mathrm{Fe})=8.3 \mathrm{~km} / \mathrm{sec}$. The bisection of a line with a cross wire to an accuracy around $0.5 \mathrm{~km} / \mathrm{sec}$ is thus feasible; repeated measurements have, indeed, shown such precision. On the assumption that the profiles to which the above widths correspond are Gaussian, we may easily disentangle the thermal and turbulent components of the mean square radial velocities, through the dependence on atomic weight of the former. We find from the representative values given above

$$
\begin{aligned}
\left\langle V^{2}\right\rangle_{\text {thermal }}^{\frac{1}{t^{2}}} & =8.9 \mathrm{~km} / \mathrm{sec} \quad(\text { for } \mathrm{H}) \\
\left\langle V^{2}\right\rangle_{\text {turb }} & =7.4 \mathrm{~km} / \mathrm{sec} .
\end{aligned}
$$

The corresponding kinetic temperature is $9700^{\circ} \mathrm{K}$, in close agreement with the value of the electron temperature determined by other methods.

Any interpretation of the observed variation in radial velocity across the nebula in terms of some hydrodynamical model, should also account for the line widths. It is precisely in this connection that von Hoerner's analysis exhibits inconsistencies. The observations show that the turbulent component of the line widths is comparable to the range of variations of velocity across the nebula inferred from line shifts. This would indicate that the effective depth of line formation $D$ is not smaller than the distance scale $\Lambda_{0}$ of the radial velocity variations provided the velocity field is isotropic and homogeneous. But if the correlation function between the observed radial velocities at two points separated by a distance $\Lambda$ varies as $\Lambda^{\frac{1}{3}}$, and this dependence is interpreted as proof of the similarity hypothesis, then necessarily the averaging along the line of sight should be negligible and $D \ll<\Lambda_{0}$. On the basis of these results alone, one could have inferred that the Kolmogoroff law could not possibly be satisfied in the nebula, unless of course the earlier observations were affected by gross errors. The excellent agreement of our observational results with the earlier measures definitely rules out an explanation of the contradiction referred to above in terms of observational errors.

Allow me to consider in some detail the meaning of the observational data we are discussing: An element $P$ of the nebula with density $\rho$ and subtending a solid angle $d \omega$ at the observer, emits energy $d E_{v^{\prime}}$ with a frequency distribution, here measured in velocity units, of the form

$$
d E_{v^{\prime}}=\frac{Q}{\delta(2 \pi)^{\frac{1}{2}}} \exp \left[-\left(v^{\prime}-V(s)\right)^{2} / 2 \delta^{2}\right] \rho^{2} s^{2} d s d \omega,
$$


where $\delta$ is the rms velocity of the Maxwellian distribution of temperature $T$ prevailing at that point, $v^{\prime}$ is the total velocity (mass motion plus thermal motion),

$$
\delta^{2}=k T / \mu m_{\mathrm{H}},
$$

$V=V(s)$ is the radial component of mass motion, and $Q$ is some parameter depending on the cross section for recombination and the relative abundance of the elements. In principle, the velocity $V(s)$ depends also on time, but the scale of the temporal variations in the velocity field is of the order of several hundred years; thus all our observations refer in practice to a particular instant. The energy received at the earth per unit solid angle and unit surface, or the specific intensity of radiation $I_{v^{\prime}}$, is then

$$
I_{v^{\prime}}=\int_{s_{1}}^{s_{2}} Q \rho^{2} e^{-\tau(s)} \exp \left[-\left(v^{\prime}-V\right)^{2} / 2 \delta^{2}\right] \frac{d s}{\delta(2 \pi)^{\frac{1}{2}}} .
$$

The weighting factor $e^{-\tau(s)}$ is introduced to take into account the extinction of light produced by the solid particles immersed in the medium. It is known that $\tau\left(s_{2}\right)$ is so large that we may set $s_{2}=\infty$ and by a suitable change of origin we choose $s_{1}=0$. To a high degree of approximation $T=$ constant, as it is determined almost entirely by radiative processes. The total intensity of the line is then

$$
A=\int_{-\infty}^{\infty} I_{v^{\prime}} d v^{\prime}=Q \int_{0}^{\infty} \rho^{2} e^{-\tau(s)} d s
$$

From Eq. (3) it follows that the velocity of the center of figure of the profile which is the measured radial velocity $\mathrm{V}$, is

$$
\mathrm{V}=\frac{Q}{A} \int_{0}^{\infty} \rho^{2} e^{-\tau(s)} V(s) d s,
$$

and the mean square width of the profile $I_{v^{\prime}}$ is

$$
\begin{aligned}
\Delta^{2}=\frac{Q}{A} \int_{-\infty}^{\infty}\left(v^{\prime}-\mathrm{V}\right)^{2} I_{v^{\prime}} d v^{\prime} & \\
& =\delta^{2}+\frac{Q}{A} \int_{0}^{\infty} \rho^{2} e^{-\tau(s)}[V(s)-\mathrm{V}]^{2} d s .
\end{aligned}
$$

From the statistical behavior of the measures $A, \mathbf{V}$, and $\Delta$, we should in principle be able to derive some information regarding $\tau(s), \rho(s)$, and $V(s)$. A further independent relation between $\tau(s)$ and $\rho(s)$ could be studied using the intensity ratio of the [OII] doublet (Seaton-Osterbrock method), but since accurate line intensity measures have not yet been carried out, we shall consider only Eqs. (5) and (6), assuming that $\rho(s)=$ constant and $\tau(s)=\kappa s$.

Let $\mathbf{V}_{\alpha}$ and $\mathbf{V}_{\beta}$ be the measured radial velocities at two points on the nebula separated by a distance $\Lambda$, and let us form the average

$u^{2}(\Lambda)=\left\langle\left(\mathbf{V}_{\alpha}-\mathbf{V}_{\beta}\right)^{2}\right\rangle_{\mathrm{Av}}$

$$
\begin{aligned}
&=\kappa^{2} \int_{0}^{\infty} \int e^{-\kappa\left(s_{1}+s_{2}\right)}\left\{\left\langle\left[V_{\alpha}\left(s_{1}\right)-V_{\beta}\left(s_{2}\right)\right]^{2}\right.\right. \\
&\left.\left.-\left[V_{\alpha}\left(s_{1}\right)-V_{\alpha}\left(s_{2}\right)\right]^{2}\right\rangle_{\mathrm{AU}}\right\} d s_{1} d s_{2},
\end{aligned}
$$

over all pairs of points $(\alpha, \beta)$ in the nebula separated in projection by the distance $\Lambda$, and along the two lines of sight intersecting the boundary of the nebula at the points $\alpha$ and $\beta$. The expected value of Eq. (6) is, similarly,

$\left\langle\Delta^{2}\right\rangle_{A V}-\delta^{2}=\frac{1}{2} \kappa^{2}$

$$
\times \int_{0}^{\infty} \int e^{-\kappa\left(s_{1}+s_{2}\right)}\left\langle\left[V_{\alpha}\left(s_{1}\right)-V_{\alpha}\left(s_{2}\right)\right]^{2}\right\rangle_{A r} d s_{1} d s_{2} .
$$

The observations provide numerical values for the quantities involved in Eqs. (7) and (8), and may then be compared with theory. Let it be supposed that the velocity variations from point to point inside the nebula are homogeneous and isotropic and that the Kolmogoroff law applies. Then we shall expect that

$$
\left.\begin{array}{l}
\left\langle\left[V_{\alpha}\left(s_{1}\right)-V_{\alpha}\left(s_{2}\right)\right]^{2}\right\rangle_{A v}=C^{2}\left|s_{1}-s_{2}\right|^{3} \\
\left\langle\left[V_{\alpha}\left(s_{1}\right)-V_{\beta}\left(s_{2}\right)\right]^{2}\right\rangle_{A v}=C^{2}\left\{\Lambda^{2}+\left(s_{1}-s_{2}\right)^{2}\right\}^{\}}
\end{array}\right\},
$$

and on this hypothesis Eq. (7) gives

$$
u^{2}(\Lambda)=C^{2} \kappa^{-\frac{3}{3}} \int_{0}^{\infty} d z e^{-z}\left[\left(l^{2}+z^{2}\right)^{\frac{1}{3}}-z^{3}\right],
$$

where we have written: $l=\kappa \Lambda$, while $z$ is an auxiliary variable. We find then the convenient form given by von Hoerner ${ }^{4}$

$$
\left.\begin{array}{l}
u(\Lambda)=C \Lambda^{1 / 3} H_{1}(l) \quad\left(\text { for } l>l_{0}\right) \\
u(\Lambda)=C \kappa^{1 / 2} \Lambda^{5 / 6} H_{1}(l) / l^{1 / 2} \quad\left(\text { for } l<l_{0}\right)
\end{array}\right\},
$$

where $l_{0}$ is an arbitrary number of order unity, and $H_{1}(l)$ is a slowly varying function of $l$ such that

$$
\left.\begin{array}{l}
H_{1}(l) \rightarrow 1 \text { as } l \rightarrow \infty \\
H_{1}(l) \rightarrow \text { const } l^{l} \text { as } l \rightarrow 0
\end{array}\right\} .
$$

In a similar fashion, from Eq. (8), we obtain

$$
\left\langle\Delta^{2}\right\rangle_{A V}-\delta^{2}=\frac{1}{2} \Gamma(5 / 3) C^{2} \kappa^{-3} \text {. }
$$

We may compare then the predictions of Eqs. (10) and (12) with the results of the observations. From our radial velocities measures of the line of [OIII], we have formed average values of $u(\Lambda)$ over samples containing several hundred pairs of points at fixed distances $\Lambda$. The resulting logarithmic dependence of $u$ on $\Lambda$ is shown in Fig. 1, where it may be seen that the representative points define a line with slope not far from the value 
$\frac{1}{3}$, in good agreement with von Hoerner's earlier results. It does not necessarily follow, however, that the observations agree with the predictions of the similarity hypothesis, for $u(\Lambda) \propto \Lambda^{\frac{1}{b}}$ only when $l=\kappa \Lambda$ is sufficiently large. Requiring this to be the case, von Hoerner estimated a value for $\kappa$ around $12 \mathrm{pc}^{-1}$, which would mean that the effective depth of the nebula is only $\kappa^{-1}=0.08 \mathrm{pc}$, a distance which on the plane of the sky is equivalent to 30 in., or about one-tenth the apparent dimensions of the bright part of the nebula. The extension of the correlation function to smaller values of $\Lambda$ by our observations would imply a further reduction of the effective depth $\kappa^{-1}$, and the resulting geometrical model for the nebula would be that of a thin sheet of material normal to the line of sight. In such a model, we would obtain from Eq. (10) $C=10 \mathrm{~km} / \mathrm{sec} \mathrm{pc}^{-\frac{1}{3}}$ and Eq. (12) would then imply a turbulent half-width $\left(\left\langle\Delta^{2}\right\rangle_{A v}-\delta^{2}\right)^{\frac{1}{2}}$ around $2.6 \mathrm{~km} / \mathrm{sec}$, a value which is three times smaller than the smallest observed turbulent component of line width!

We shall now make a direct estimate of the effective depth of the nebula and show, thus, that the large value of $\kappa$ suggested by the preceding considerations is inadmissible. The luminosity of the nebula derives from the ultraviolet radiation of the high temperature exciting stars, through the operation of the Zanstra fluorescence process. That these stars are imbedded in the nebula and not in front of it, as supposed by von Hoerner, is shown most clearly by the appearance in their spectrum of the $2^{3} S-3^{3} P$ line of HeI in absorption. The Doppler shifts of this line corresponds more closely to the velocity of the nebular lines than to the velocities of the stars concerned (see Fig. 2). When it is recalled, moreover, that the $\mathrm{HeI}$ absorption line has been observed only in two other stars exciting an HII region (M8), approaching the Orion Nebula in extension and density, it would seem unquestionable that the line

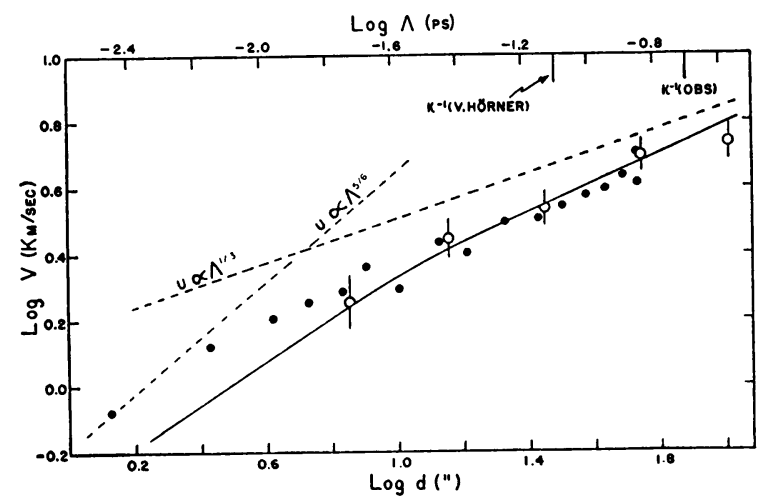

Fig. 1. Correlation between the root-mean-square difference in velocity at two points of the Orion Nebula and their mutual distance. Values derived from new observations are indicated by filled circles. Points entered as open circles are the values derived by Von Hoerner from the Lick Observatory measures, and the lines across them indicates their uncertainty, according to the same author. The curve and its asymptotes are the theoretical values fitted by von Hoerner.

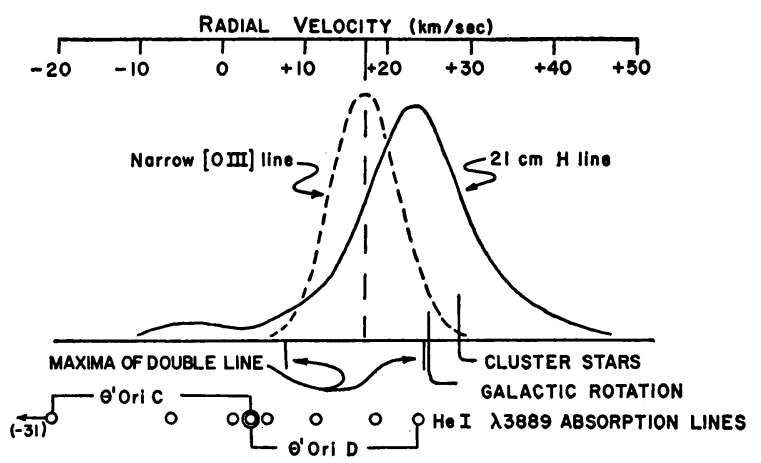

FIG. 2. Heliocentric radial velocities of the emission lines of [OIII] in the Orion Nebula, of the $21-\mathrm{cm}$ line emitted by the surrounding HI region, of the nebular absorption line of HeI on the cluster stars and of these stars themselves.

is formed in the nebula. We may estimate the optical depth of the continuous absorption of the nebula from the reddening of the stars imbedded in it. The mean color excess of the Trapezium stars is ${ }^{5}$

$$
E_{y}=+0 .{ }^{m} 40
$$

while other stars in the same association but not within the nebula have a mean color excess around $+0 .^{m} 10$. The total absorption for visual light corresponding to the difference of color excesses is then $6 \Delta E_{y}=+1 .^{m} 8$, or $\tau^{*}=\left(6 \Delta E_{y} / 1.086\right)=1.67$. If the dimension of the nebula in the radial direction is identified with the geometrical depth $S^{*}$ of the exciting stars and is assumed equal to the radius of its brightest part (about $2^{\prime}$ ) then $S^{*}=0.32 \mathrm{lpc}$ and $\kappa \approx 5 \mathrm{pc}^{-1}$. Referring again to Fig. 1 we may see that if this "observed" value of $\kappa$ is adopted, all the observed points in the correlation function would fall in the range of $\Lambda$ where the universal equilibrium theory, for that value of $\kappa^{-1}$, would predict a variation according to $\Lambda^{5 / 6}$. Since the observed points define a much less steep variation it would follow that the fundamental hypothesis of that theory is not satisfied in the Orion Nebula.

Our observations directly reveal the reasons for the the failure of the theory of incompressible turbulence to account for the state of motion in the nebula. The sudden appearance in certain regions of distinctly double lines suggest immediately the existence of discontinuities in the flow, which must be produced by shock waves. Since the velocity of sound in the nebula is around $12 \mathrm{~km} / \mathrm{sec}$ and the apparent splitting of the lines in places amounts to $25 \mathrm{~km} / \mathrm{sec}$, it is clear that the compressibility effects on the motions of the medium will be of primary importance, while shear turbulence and vortex motion will be secondary. It may be of interest to describe briefly here the observed characteristics of the line doubling, as it may give clues for the complete understanding of the phenomena involved.

(a) The transition from a spot where the line is

${ }^{5}$ S. Sharpless, Astrophys. J. 116, 251 (1952). 
narrow to one where it appears double often takes place over distances only of the order of a few seconds of arc. The dimensions of the regions where the lines may be seen as double are also small compared with the dimensions of the nebula, say of the order of a few times $10 \mathrm{sec}$ of arc. The position of the areas with line doubling does not have any obvious relation to the geometry of the nebula and exciting stars as a whole. There may be some connection between the line doubling and changes in surface brightness, but we are not in a position yet to make this correlation precise (see Fig. 3).

(b) The intensity ratio between the components of a double line varies appreciably over the region where it is observed as such. The apparent velocities of the components generally differ from the velocities where it is measured as single. That is to say, the doubling is not of the nature of one line staying more or less at the same velocity and developing a satellite only in a small area. We are certain that this is not an instrumental effect, because at some places where the [OIII] line is double the [OII] cannot be seen as such (see Fig. 4), but its velocity follows that of one of the displaced [OIII] components.

(c) The different behavior of the [OII] and [OIII] lines reveals immediately the existence of large differences between the physical conditions (temperature and density) prevailing in the two masses whose relative motion we are observing. In most cases the violet

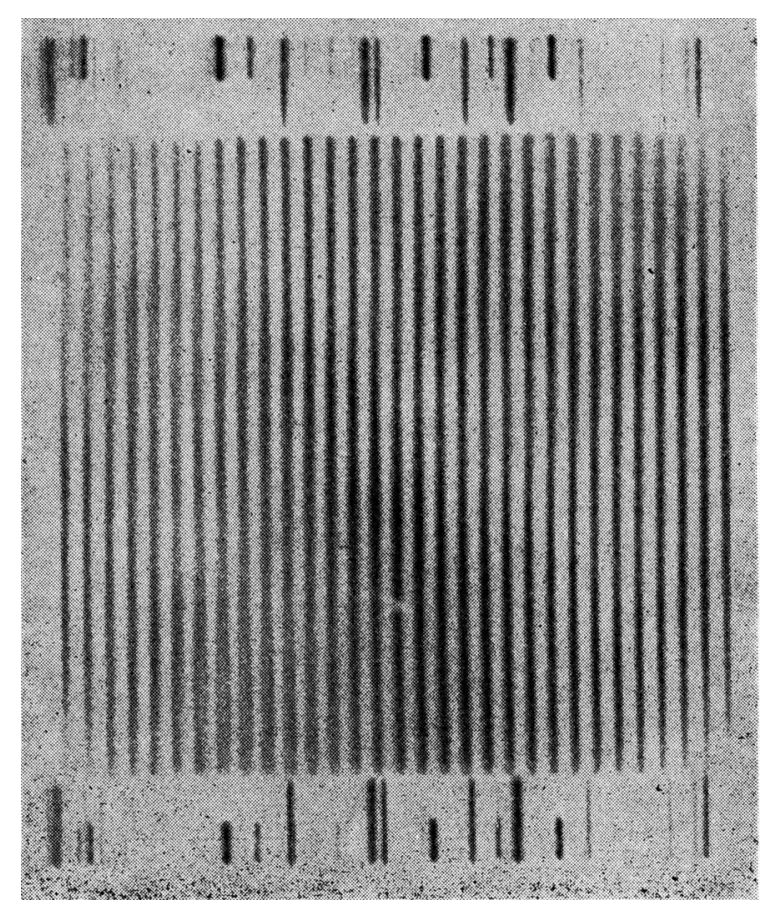

FIG. 3. Multislit [OIII] image of an area of the Orion Nebula with center at $22^{\prime \prime} .5 \mathrm{E}$ and $16^{\prime \prime} .3 \mathrm{~S}$ of $\theta^{1}$ Ori $\mathrm{C}$, and dimensions $35^{\prime}$ in the E-W direction (along the slits) and $41^{\prime}$ in the N-S direction (along dispersion). The image is oriented with east at top and north at right. displaced component (approaching) of the [OII] line is weaker than the corresponding [OIII] line. We are certain that this effect is a consequence of increased ionization and not of differential continuous absorption, because the $[\mathrm{NeIII}]$ line at $\lambda 3869$, which should be affected by continuous absorption nearly in the same manner as the [OII] line, behaves like the [OIII]. We expect to make a direct measurement of the densities in the masses producing the two components, by measuring the intensity ratio of both lines in the [OII] doublet for both components.

The existence of discrete gas masses inside the nebula moving with relative velocities in excess of the velocity of sound is also suggested by the complex structure of the HeI $\lambda 3889$ absorption line in the spectrum of the Trapezium stars ${ }^{6}$ (see Fig. 2).

On the basis of the ideas currently held for the dynamical interaction between the high temperature gas in the nebula and the surrounding cold matter, it is not difficult to visualize, in general terms, a possible mechanism which may give origin to a system of pressure waves rushing about in all directions through the nebula. The pressure gradient existing across the boundary between the HII and HI regions causes an expansion of the hot gas into the surrounding matter. At the Cambridge Symposium there was some discussion whether the Orion Nebula was expanding at all. On the basis of our observations the expansion would seem obvious, as the nebular lines on the average show a velocity of approach of about $12 \mathrm{~km} / \mathrm{sec}$ with respect to the exciting stars and of around $6 \mathrm{~km} / \mathrm{sec}$ with respect to the surrounding cold matter, as shown by the $21-\mathrm{cm}$ line profile in Fig. 2. The motion of the ionization front into the cold region is undoubtedly quite unstable, because the density of the driver gas is less than that of the driven gas ("dynamical" Rayleigh-Taylor instability). Since the density and pressure variations in the cold gas must be large, as a consequence of the low velocity of sound $(1 \mathrm{~km} / \mathrm{sec})$ and relatively high $\mathrm{rms}$ random velocity $(8 \mathrm{~km} / \mathrm{sec})$, we should expect that masses of cold material will often be left behind the ionization front, moving inwards. The ionization of the surface layers of such dense pockets of cold gas by radiation from the stars, as it advances into the lighter hot medium, will produce large dynamical effects. Depending on the relative densities and relative velocities of the hot and cold masses, compression waves will necessarily develop through one or both media. This mechanism is essentially that invoked by Oort and Spitzer ${ }^{7}$ to convert energy of radiation of the hot stars into kinetic energy of the cold interstellar medium. Details of this process in particular idealized cases have been worked by ${ }^{6}$ W. S. Adams, Publs. Astron. Soc. Pacific 56, 119 (1944).
(Also O. C. Wilson, unpublished.)

${ }_{7}$ J. H. Oort and L. Spitzer, Astrophys. J. 121, 6 (1955). 


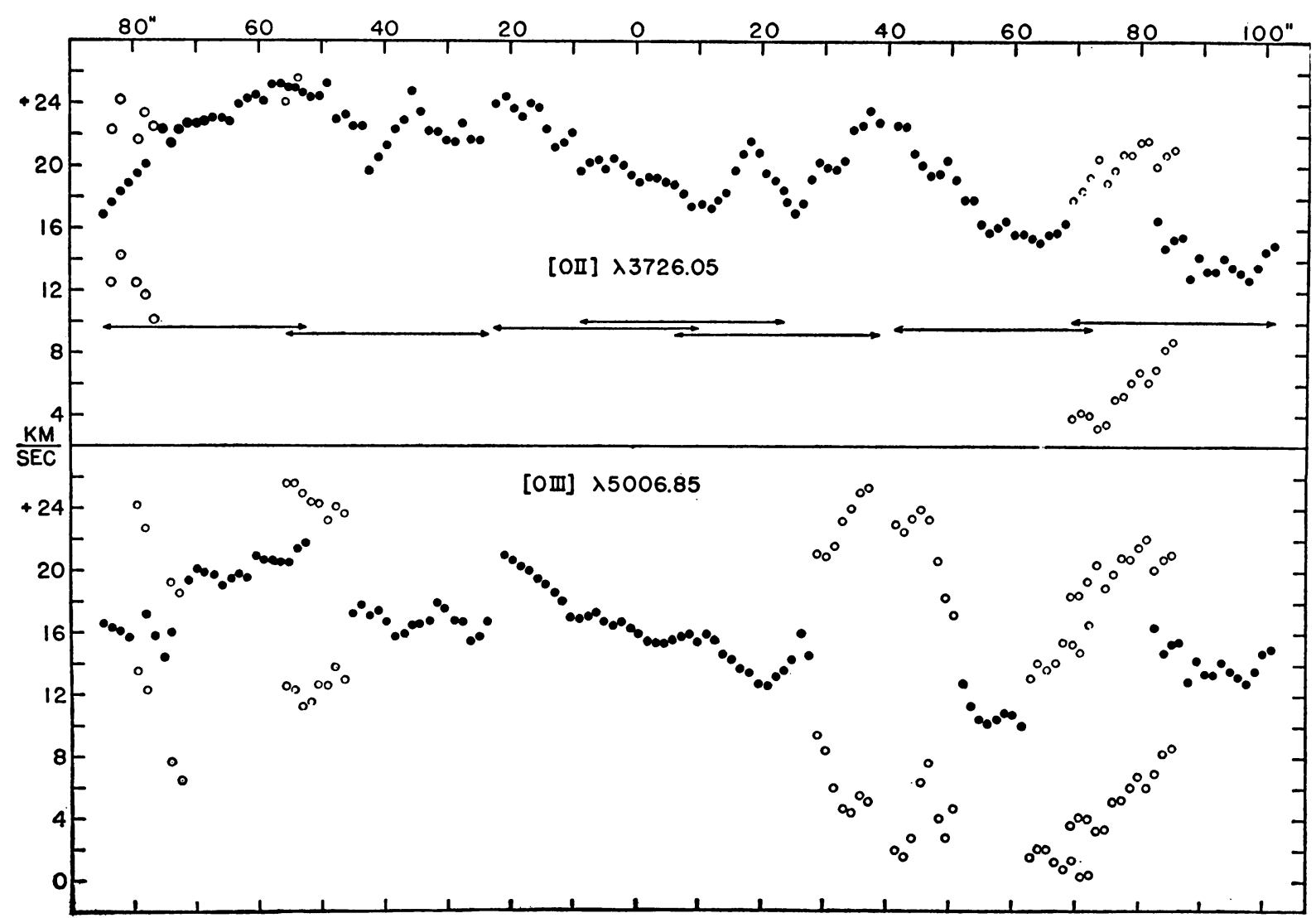

FIG. 4. Radial velocities of the [OII] and [OIII] lines in the Orion Nebula along an E-W line, 25 in. S of $\theta^{1}$ Ori C. Empty circles indicate points at which lines are measured as double. The extent of the nebula covered by one plane is indicated by the lines limited by arrows appearing in the graph for the [OII] line.

$\mathrm{Kahn}^{8}$ and Savedoff and Greene, ${ }^{9}$ but so far more attention has been given to what happens to the cold medium than to the dynamical effects which may arise in the hot gas.

In conclusion we may inquire into the possible meaning of the empirical correlation of velocities exhibited earlier in Fig. 1. Taken at face value it implies that the velocities at two points within the nebula are much more strongly correlated than if the dissipation

${ }^{8}$ F. D. Kahn, Bull. Astron. Soc. Neth. 12, No. 456, 187 (1954).

${ }^{\circ}$ M. P. Savedoff and J. Greene, Astrophys. J. 122, 477 (1955). of the kinetic energy of mass motion were taking place only at the smallest eddies, as in incompressible turbulence. It was emphasized by several participants to the Cambridge Symposium, (Biermann, Kantrowitz, Lighthill), this is precisely what one would expect to happen in compressible turbulence: there is a direct transfer of energy from the large scale elements, to which the energy is being fed, to the regions where the dissipation is taking place (the shock fronts), with the consequence that elements with intermediate wave numbers are much suppressed.

\section{DISCUSSION}

M. MINNAERT, Sterrewacht Sonnenburg, Utrecht, Netherlands: How good are the new results, when compared with the old measurements of Baade and Minkowski obtained with an interferometer?

G. MÜNCH, California Institute of Technology, Pasadena, California: The Baade and Minkowski measurements have rather low angular resolving power; thus, they are integrated over areas of the nebula much larger than those we measured. However, if one picks an area where there is no doubling, our results agree very well.

S. B. PICKelner, Crimean Astrophysical Observatory, Simeis, U.S.S.R.: I should like to recall a paper written several years ago by Shajn and myself on the character of the movements in the Orion Nebula. We photographed the central part of the Orion Nebula in $\mathrm{H} \alpha$, [OIII], and [OII]. The surface brightness fluctuations may be considered as an index of the 

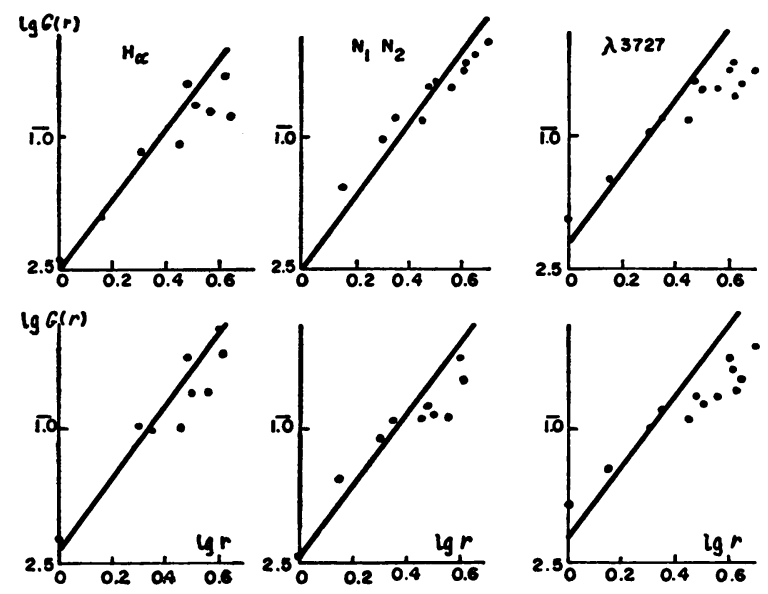

FIG. 1.

character. of the internal motion. From the observations, we can calculate the statistical function which characterizes the brightness fluctuation

$$
G(r)=\frac{\left\langle\left[J\left(m_{i}\right)-J\left(m_{i}{ }^{\prime}\right)\right]^{2}\right\rangle_{\mathrm{Av}}}{\left\langle J^{2}\right\rangle_{\mathrm{Av}}},
$$

where $m_{i}$ and $m_{i}{ }^{\prime}$ represent a pair of points at distance $r$.

The expression for the corresponding theoretical function was obtained on the assumption that locally isotropic turbulence is present with speeds lower than the speed of sound. It is supposed that the emission is proportional to $p^{2}$, where $p$ is the pressure; with $p_{1}$ and $p_{2}$ representing the pressures at two points separated by distance $r$, and $v_{1}$ and $v_{2}$ representing the velocities; we have assumed

$$
\begin{aligned}
\left\langle\left(p_{1}-p_{2}\right)^{2}\right\rangle_{\mathrm{AV}} & =C^{2} \rho^{2} \epsilon^{\mathrm{k} r^{3}}, \\
\left\langle\left(v_{1}-v_{2}\right)^{2}\right\rangle_{\mathrm{AV}} & =C^{2} \epsilon^{3} r^{3},
\end{aligned}
$$

where $C$ is a constant of order unity, while $\epsilon$ is the dissipation of turbulent energy in ergs per gram and per second. After a series of transformations common in the theory of turbulence, we obtain a correlation function $F(r)$ of the type,

where

$$
F(\boldsymbol{r})=C^{2} m_{\mathrm{H}^{2}}(k T)^{-2}(\epsilon r)^{\mathbf{t}} \varphi(r / l),
$$

$$
\varphi(r / l)=(r / l) \int_{0}^{\infty} \exp (-r u / l)\left[\left(1+u^{2}\right)^{2 / 3}-u^{4 / 3}\right] d u .
$$

Here $l$ is the size of the layer, the optical thickness of which is equal to unity. The function $\varphi(r / l)$ has been calculated numerically; if $r / l \geq 0.5, \varphi(r / l)$ is about equal to 1.

The results of the calculation are shown in Fig. 1 for different spectral lines. The bottom row refers to the central part of the nebula, and the upper row to a larger region. It is seen that the values of $G(r)$ coincide with the straight lines, the slope of which is $\frac{4}{3}$; but for [OIII] and for the larger values of $r$, the slope of the curves is less. From these curves we determined $C^{\frac{3}{2}} \epsilon$ in $\mathrm{erg} / \mathrm{g} \mathrm{sec}$, which came out approximately equal to 1 , in good accordance with the values obtained by von Hoerner from the discussion of radial velocities. The size of the emitting layer was calculated by means of the Kolmogoroff formula, using this value of $\epsilon$ and the mean dispersion of the radial velocities derived by Baade, Goos, Koch, and Minkowski (about $6.2 \mathrm{~km} / \mathrm{sec}$ ). The thickness of the layer is about $0.1 \mathrm{pc}$; and the absorption is about $10 \mathrm{~m} / \mathrm{pc}$. This value is in accordance with the color excess of $\theta^{\prime}$ Orionis, if this star is situated in the center of the nebula.

There is a point of interest in a drawing given in Baade's paper which was not used by the authors. The drawing presented the intensity and the velocity dispersion along two cross sections, and showed that the dispersion of the radial velocity is larger at those points where the brightness is smaller. An interpretation may be connected with the turbulent character of the motions. The greater brightness of the point corresponds to the greater density of the gas, because the emission of the gas is proportional to $\rho^{2}$. The density of the dust will also be larger at this point, and the layer will be thinner here, the thickness being proportional to $\rho^{-1}$. Thus, the thickness is less if the brightness is larger. The low-velocity dispersion in these points means that the principal property of the turbulent motion - the increase of the velocity with the scale-is fulfilled in the Orion Nebula. It is not necessarily the pure Kolmogoroff law, but the larger scale has a larger velocity difference.

H. W. LIEPMANN, California Institute of Technology, Pasadena, California: From the point of view of turbulence, we have discussed now two effects which we should not confuse. One was the distance effect, taking account of the fact that the measurements are averages over certain depths. The other effect is compressibility; density fluctuations were, of course, excluded in the original Kolmogoroff work. What we discuss now are observations averaged in depth on compressible turbulence. There is really no reason why we should expect the Kolmogoroff spectrum. If I understand Pickelner rightly, at least the character of the spectrum is such that it looks like turbulence plus a density fluctuation, i.e., something of the nature of compressible turbulence, and this follows also from Münch's paper.

Bertram Donn, Wayne State University, Detroit, Michigan: The occurrence of multiple emission lines in Orion and Münch's interpretation of them is, on a small scale, essentially the same picture as I proposed for the behavior of interstellar gas on the galactic scale. This is the existence of high velocity currents among general density fluctuations. Why does the explanation work here, but not for the case of multiple absorption components which you used as an argument against such currents? 
G. MÜNCH : I think the answer is very simple. In the case of the cool gas, the velocity of sound is of the order of $1 \mathrm{~km} / \mathrm{sec}$, and here it is about $15 \mathrm{~km} / \mathrm{sec}$.

BERTRAM DONN : I was referring principally to the interpretation of the observations. When you showed the slide following my paper, you said that on the basis of that slide you don't see how you could have separate clouds, whereas here you are willing to grant separate moving elements which are close to each other.

G. MÜNCH : I think, in both cases, I am trying to say that there are separate gas masses moving with different velocities and which do not have continuity between them. I do not really see any difference.

M. P. SAVEDOFF, University of Rochester, Rochester, New York: All discussions of the energy of the inter- stellar medium so far have been directed toward the motion of the cool gases. Münch has now raised a new problem, how to get the energy into the hot gas.

G. MÜNCH : I believe that a mass of hot gas can be stirred up to a state of random motion at higher speeds only through interaction with a cold gas. There is no way in which radiation from the hot stars can get hold of the hot gas. I would make the guess that that is the reason why the gas in planetary nebulae are not turbulent; we observe that the lines are sharp.

H. W. LIEPMANN : From the figure which I saw on Münch's slide, the ratio of the turbulent velocity to the velocity of sound is nearly one, a result which makes a great deal of sense, since any motion faster than that would be more rapidly damped. 